\title{
Microscopic Dynamics of Liquid-Liquid Phase Separation and Domain Coarsening in a Protein Solution Revealed by X-Ray Photon Correlation Spectroscopy
}

\author{
Anita Girelliø, ${ }_{1}^{1}$ Hendrik Rahmann, ${ }^{2}$ Nafisa Begam $\odot,{ }_{1}^{1}$ Anastasia Ragulskaya, ${ }_{1}^{1}$ Mario Reiser, ${ }^{2,3}$ Sivasurender Chandran $\odot,{ }^{1,4}$ \\ Fabian Westermeier $\odot,{ }^{5}$ Michael Sprung, ${ }^{5}$ Fajun Zhang $\odot,{ }^{1, *}$ Christian Gutt $\oplus^{2, \dagger}$ and Frank Schreiber $\oplus^{1, *}$ \\ ${ }^{1}$ Institut für Angewandte Physik, Universität Tübingen, Auf der Morgenstelle 10, 72076 Tübingen, Germany \\ ${ }^{2}$ Department Physik, Universität Siegen, Walter-Flex-Strasse 3, 57072 Siegen, Germany \\ ${ }^{3}$ European X-Ray Free-Electron Laser XFEL, Holzkoppel 4,22869 Schenefeld, Germany \\ ${ }^{4}$ Department of Physics, Indian Institute of Technology Kanpur, Uttar Pradesh 208016, India \\ ${ }^{5}$ Deutsches Elektronen-Synchrotron DESY, Notkestraße 85, 22607 Hamburg, Germany
}

(Received 19 October 2020; accepted 23 February 2021; published 2 April 2021)

\begin{abstract}
While the interplay between liquid-liquid phase separation (LLPS) and glass formation in biological systems is highly relevant for their structure formation and thus function, the exact underlying mechanisms are not well known. The kinetic arrest originates from the slowdown at the molecular level, but how this propagates to the dynamics of microscopic phase domains is not clear. Since with diffusion, viscoelasticity, and hydrodynamics, distinctly different mechanisms are at play, the dynamics needs to be monitored on the relevant time and length scales and compared to theories of phase separation. Using x-ray photon correlation spectroscopy, we determine the LLPS dynamics of a model protein solution upon low temperature quenches and find distinctly different dynamical regimes. We observe that the early stage LLPS is driven by the curvature of the free energy and speeds up upon increasing quench depth. In contrast, the late stage dynamics slows down with increasing quench depth, fingerprinting a nearby glass transition. The dynamics observed shows a ballistic type of motion, implying that viscoelasticity plays an important role during LLPS. We explore possible explanations based on the Cahn-Hilliard theory with nontrivial mobility parameters and find that these can only partially explain our findings.
\end{abstract}

DOI: 10.1103/PhysRevLett.126.138004

Recent work suggests that structure formation in biology can take place, inter alia, through liquid-liquid phase separation (LLPS) [1-3]. Phase separation in crowded environments thus represents a mechanism for intracellular organization via the formation of biomolecular condensates [4]. The biological functions of these condensates-including steering biochemical reactions rates, sensing, or signaling — are being intensely investigated [3]. LLPS is also associated with a variety of diseases caused by a loss and/or change of function of the condensates $[5,6]$.

The state of the condensates depends on the dynamic processes during their formation, often involving nonequilibrium processes over a hierarchy of length and time scales [2,7]. A case in point is the slowdown of the dynamics on molecular length scales caused by concentration and its influence on the dynamical and structural properties of the condensate on mesoscopic length scales. Ultimately, such a microscopic slowdown can lead to the arrest of LLPS on larger length scales accompanied by the formation of bicontinuous gel network structures [8-10].

The kinetics of arrested phase separations in protein solutions have been studied successfully in the past, demonstrating that the ensemble-averaged structure factor ceases to develop further in $q$ position and intensity upon low temperature quenches [11-15]. However, the dynamics of protein solutions en route to an arrested LLPS is largely unknown, mainly because of the requirement to monitor an exceptionally broad range of time and length scales simultaneously. This, in turn, prevented the experimental validation of models of the dynamics of critical phenomena during LLPS, such as the Cahn-Hilliard equation and related models, especially in the vicinity of glass-gel transitions displaying large dynamical asymmetries between the species involved [2].

LLPS is a general phenomenon, which is relevant not only for protein systems but also in many other fields of science [16]. LLPS domains have been studied with different microscopy techniques [17,18]. Their macroscopic properties such as turbidity and viscosity [19-21] were monitored, as well as their molecular properties [20,22-27]. Other scattering techniques were used to access the kinetics of the phase separation [14,15,19,21].

X-ray photon correlation spectroscopy (XPCS) employing coherent $\mathrm{X}$-rays can resolve the collective dynamics on the required length scales, ranging simultaneously from nanometers to microns and timescales from microseconds to hours [28-35]. Here, we demonstrate that a combination of scanning techniques, large beams, and long sample detector distances [36-38] allows us to reduce the required $\mathrm{x}$-ray doses to values below the critical dose of many 
protein systems [39]. More details on the experimental parameters are provided in the Supplemental Material (SM) [40]. With this approach, we are able to follow the dynamics during an LLPS of $\gamma$ globulin (Ig) in a concentrated aqueous polyethylene glycol (PEG) solution. The experimental results are compared to simulations based on the Cahn-Hilliard equation, taking the gel transition into account (in the spirit of model C according to Ref. [2]; see the SM) $[2,45,46]$. Our work paves the way for future XPCS experiments exploring the full spatiotemporal window of LLPS, allowing one also to benchmark and guide computer simulations of complex protein dynamics in crowded environments.

XPCS experiments were conducted at the P10 Coherence Applications Beamline at PETRA III, Deutsches ElectronenSynchrotron, employing an x-ray beam of photon energy $8.54 \mathrm{keV}$, a size of $100 \times 100 \mu \mathrm{m}^{2}$, and a maximum photon density of $10^{7}$ photons $/ \mathrm{s} / \mu \mathrm{m}^{2}$. The key for performing low dose XPCS experiments is to make use of large beams with a sufficient degree of coherence. Time series of coherent diffraction patterns were collected with an EIGER 4-megapixel detector covering a $q$ range from 0.003 to $0.05 \mathrm{~nm}^{-1}$. Samples of Ig with PEG and $\mathrm{NaCl}$ have been prepared as described in [15] and in the SM and quenched from $37^{\circ} \mathrm{C}$ to different quench temperatures $T_{q}$ below the binodal line.

Figure 1(a) displays the temporal evolution of the scattering intensity as a function of scattering vector $q$ for a quench temperature of $T_{q}=10^{\circ} \mathrm{C}$ [for other quench temperatures, see Figs. S1(a) and S1(b) in the SM], capturing the LLPS process during the first $60 \mathrm{~s}$. The $\mathrm{x}$-ray intensity increases rapidly during the early time of the phase separation, and the position of the spinodal peak $q_{\max }$ shifts to smaller values, indicating an increase in length scales of the concentration fluctuations. Appropriately normalizing the intensity and wave vectors by the respective peak intensities $I\left(q_{\max }\right)$ and positions $q_{\max }$, we obtain a master plot as expected for spinodal decomposition [47]
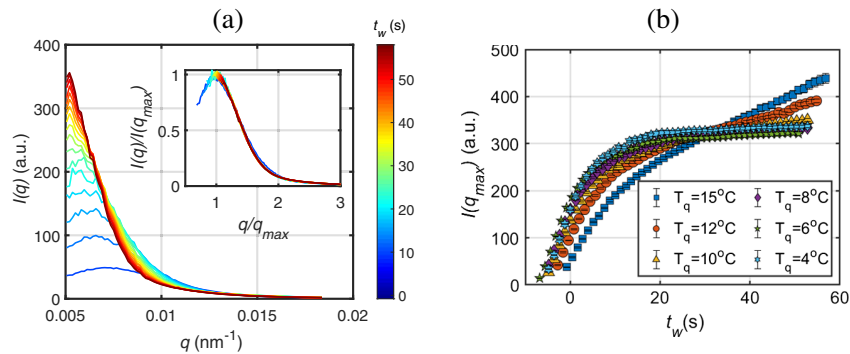

FIG. 1. (a) Intensity as a function of scattering vector $q$ for a quench temperature $T_{q}=10^{\circ} \mathrm{C}$; in the inset, the rescaled intensity. The different colors correspond to different waiting times $t_{w}$ as indicated in the color bar. The time $t_{w}=0$ is the time at which the quench temperature $T_{q}$ was reached. (b) The intensity at the peak position as a function of time for different temperatures. The error bars are within the symbol size if they are not visible. [see inset, Fig. 1(a) and Figs. S1(c) and S1(d)]. $I\left(q_{\max }\right)$ shows a rapid increase during the early stage, with a rate determined by the quench depth, while at around $10 \mathrm{~s}$ the growth starts to slow down considerably [Fig. 1(b)]. This slowdown is more pronounced when quenched to lower temperatures, while at higher temperatures, $I(q)$ continues to grow even beyond $40 \mathrm{~s}$, albeit at a slower rate. Insights into the dynamics during the LLPS are obtained by analyzing a time series of the coherent $x$-ray speckle patterns. For this, we calculate two-time correlation functions (TTCs) for specific scattering vectors $q$ and different quench depths via

$$
C\left(t_{1}, t_{2}, q\right)=\frac{\left\langle\left[I\left(t_{1}\right)-\bar{I}\left(t_{1}\right)\right]\left[I\left(t_{2}\right)-\bar{I}\left(t_{2}\right)\right]\right\rangle}{\sqrt{\left[\bar{I}^{2}\left(t_{1}\right)-\bar{I}^{2}\left(t_{1}\right)\right]\left[\bar{I}^{2}\left(t_{2}\right)-\bar{I}^{2}\left(t_{2}\right)\right]}},
$$

with $\langle\cdot\rangle$ being an average over detector pixels corresponding to a specific range $q \pm \delta q$ (calculated for $q$ from 5 to $11 \mu \mathrm{m}^{-1}$ and $\delta q=0.3 \mu \mathrm{m}^{-1}$ ) and $\bar{I}\left(t_{1}\right)=\left\langle I\left(t_{1}\right)\right\rangle$. From this quantity, the time dependent $g_{2}\left(t, t_{w}, q\right)=C\left(t+t_{w}, t_{w}, q\right)$ have been extracted by horizontal cuts along $t_{1}$, starting at the diagonal of the respective TTC [48].

Figure 2 shows in the upper panel the TTCs and in the lower panels the correlation function $g_{2}\left(t, t_{w}, q\right)$ for quench temperatures of $T_{q}=15,8$, and $4{ }^{\circ} \mathrm{C}$ (from left to right), respectively. We identify three stages of the dynamics during LLPS: the first stage appears directly after the quench in the first $20 \mathrm{~s}$, and its very fast dynamics is visible only by a thin line in the TTC and by the final part of the decay in the correlation function. After this early stage, the TTC shows a pronounced slowing down of the dynamics accompanied by a rising background level visible by the appearance of a squarelike feature. This corresponds to a second relaxation mode with a much slower relaxation time appearing in the $g_{2}$ functions. The contribution of this relaxation channel to the overall decay is increasing rapidly. After ca. $40 \mathrm{~s}$, the fast process in the LLPS is coming to an end, as evidenced by the disappearance of the first decay.
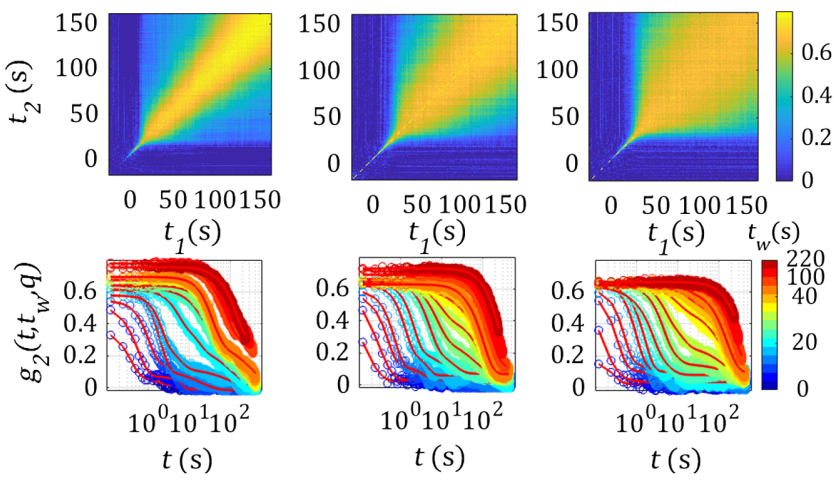

FIG. 2. In the upper panels, the two-time correlation function for $T_{q}=15,8$, and $4{ }^{\circ} \mathrm{C}$ (left to right) at $q=0.005 \mathrm{~nm}^{-1}$ are displayed, and the lower panels show the corresponding $g_{2}$ functions at different waiting times $t_{w}$. 
(a)

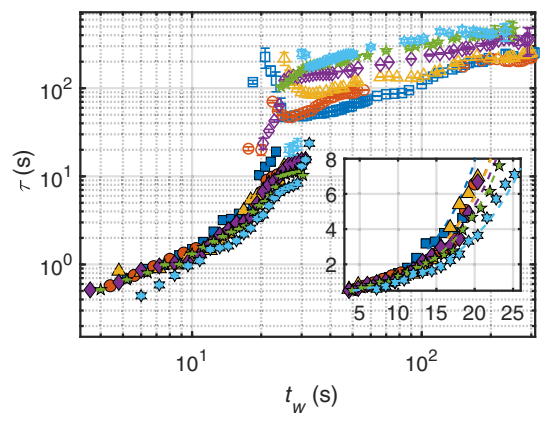

(b)

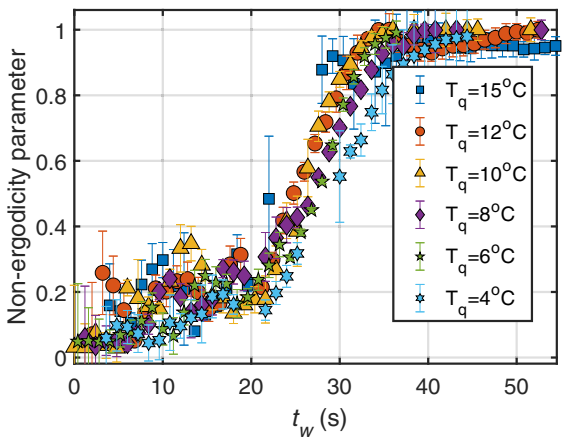

(c)

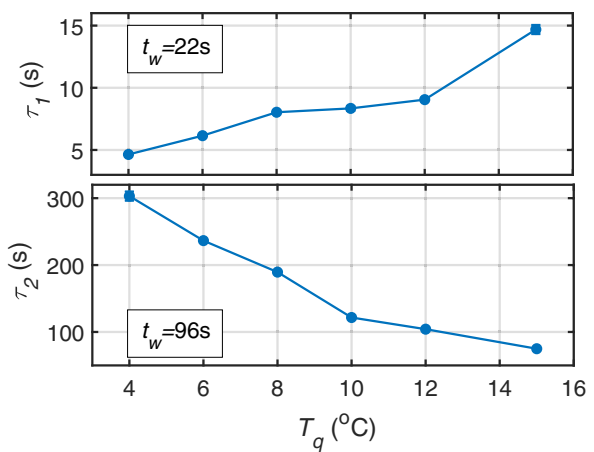

FIG. 3. (a) Decorrelation time for $q=0.005 \mathrm{~nm}^{-1}$ as a function of waiting time $t_{w}$. The open symbols correspond to the slow mode $\left(\tau_{2}\right)$ and the filled symbols to the fast one $\left(\tau_{1}\right)$. Inset: linear representation. (b) Nonergodicity parameter $f=A_{2} /\left(A_{1}+A_{2}\right)$ as a function of $t_{w}$. The legend also applies to (a). (c) Decorrelation time of the fast and slow modes as a function of quench temperature $T_{q}$, respectively.

In the third stage, later also called the late stage, the TTC evidences a second slowing down process, with the relaxation time depending on the final quench temperature with slower dynamics visible for lower temperatures (from $T_{q}=15^{\circ} \mathrm{C}$ to $T_{q}=4{ }^{\circ} \mathrm{C}$, on the top row $)$. The $g_{2}\left(t, t_{w}, q\right)$ functions have been fitted by a sum of KohlrauschWilliams-Watts (KWW) functions [49], resulting in the following equation:

$g_{2}\left(t, t_{w}, q\right)=A_{1} \exp \left[-\left(2 \frac{t}{\tau_{1}}\right)^{\gamma_{1}}\right]+A_{2} \exp \left[-\left(2 \frac{t}{\tau_{2}}\right)^{\gamma_{2}}\right]$

with decorrelation times $\tau_{1}$ and $\tau_{2}$, relaxation amplitudes $A_{1}$ and $A_{2}$, and KWW exponents $\gamma_{1}$ and $\gamma_{2}$. We note that for some $g_{2}$ functions a third exponential decay with small amplitude and slow decay is needed to describe additional tails in $g_{2}$ at very long timescales. However, due to the low statistics of the third decay, we evaluate only the two leading decays here.

Figure 3(a) displays the relaxation times as a function of waiting time $t_{w}$. We identify an exponential increase of the relaxation time in the early stage of the LLPS and faster dynamics with increasing quench depths [see Fig. 3(c) and inset of Fig. 3(a)]. At this early phase of the spinodal decomposition, the dynamics is driven by the curvature of the free energy $\partial^{2} F / \partial c^{2}$, which increases in magnitude with deeper quenches (lower temperatures) and thus speeds up the dynamics. This picture reverses when the slow relaxation sets in $\left[\tau_{2}\right.$ in Fig. 3(c)]. Now the relaxation times for the lower temperatures are considerably larger than the high temperature quenches.

The transition from dynamics dominated by the fast decay to dynamics dominated by the slow decay is quantified by the nonergodicity parameter [50], here defined as $f=A_{2} /\left(A_{1}+A_{2}\right)$. A rather sharp increase of the nonergodicity parameter around $t_{w}=30 \mathrm{~s}$ is observed
[Fig. 3(b)], with a later rise time and slower increase for lower temperatures, suggesting that the transition to coarsening dynamics is already slowed down by the lower mobility at low temperatures.

These three stages in the dynamics can be linked to the evolution of the LLPS. The first corresponds to the early stage of a spinodal decomposition, displaying an enhancement of the concentration fluctuations and the formation of an interface between two different phases. The third one is the late stage in which the domains are growing. For our system, this seems to be controlled by the mass transport mechanism and by the mobility of the involved components. In other cases in condensed matter physics, different domain growth mechanisms have been identified [51]. The second stage is a transition between the early and late stage.

Important for the assessment of the form of the dynamics and relevant for computing quantities such as diffusion and transport coefficients during the LLPS are the relaxation rate $\Gamma(q)=1 / \tau(q)$ and the $\mathrm{KWW}$ exponents, including their $q$ dependence [32]. We find in the early phase of the LLPS a linear relationship $\Gamma \propto q$ [Fig. S2(a)] and $\gamma_{1}$ around 1.4 to 1.7 (Fig. S2) with no pronounced $q$ dependence. In contrast, the dynamics during the late coarsening stage also displays a linear $(\Gamma \propto q)$ behavior but now with a pronounced $q$ dependence of the KWW exponents, which are decreasing from values of $\gamma_{2}=2$ at small $q$ values to $\gamma_{2}=1$ at large values of $q$ [Fig. S2(d)]. Our results clearly show that the coarsening dynamics of the protein droplets at this length scale are not governed by Brownian dynamics but instead by a ballistic and partially cooperative motion of the protein droplets driven by the spinodal decomposition. Similar super diffusive ballistic types of dynamics have been observed frequently in soft matter systems, e.g., in the late stage of colloidal gelation processes [52-54], during spinodal decomposition of colloidal systems [27], and in the framework of MD simulations of metallic glasses [55] with strongly interconnected clusters moving together. Phenomenological models of single microcollapse events 
(a)

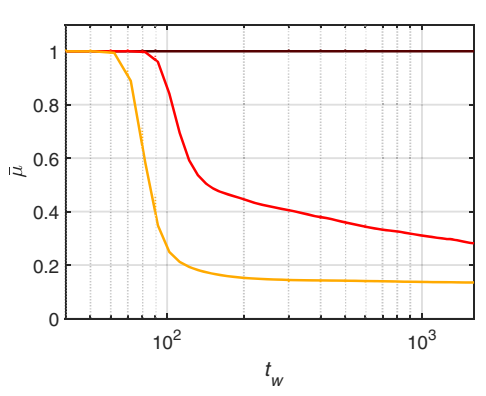

(b)

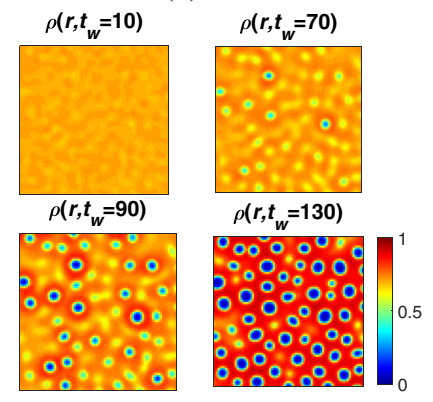

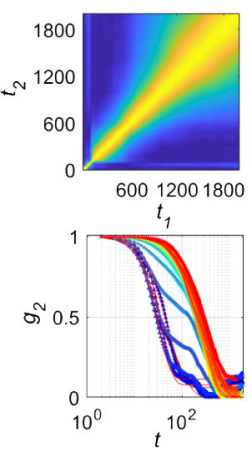

(c)

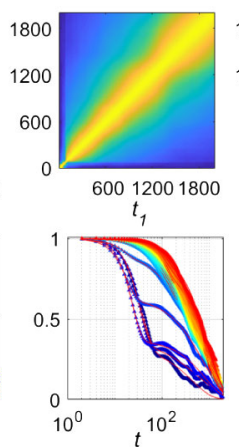

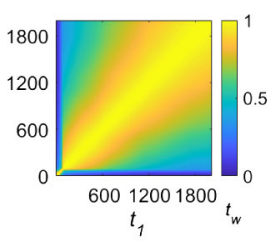

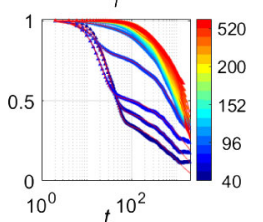

FIG. 4. The simulated $\bar{\mu}$ of the dense phase (a) as a function of waiting time. In brown, the case with constant mobility and the quench depth for this case was set to $T_{q}=0.2 \mathrm{~T}_{c}$, with $T_{c}$ being the critical temperature [62,63]. In red, $c_{g}=0.8$ and $T_{q}=0.12 \mathrm{~T}_{c}$, and in yellow, $c_{g}=0.6$ and $T_{q}=0.08 \mathrm{~T}_{c}$. (b) Examples of real space configurations for $T_{q}=0.14 \mathrm{~T}_{c}$ and mobility at different waiting times with the densities indicated by the color bar. (c) Upper panel: two-time correlation function of the simulated data for $q=1$. From left to right, the parameters $T_{q}$ and $c_{g}$ of the simulations are $T_{q}=0.2 \mathrm{~T}_{c}$ and $c_{g}=\infty$, which correspond to a constant mobility set to 1 , $T_{q}=0.12 \mathrm{~T}_{c}$ and $c_{g}=0.8$, and $T_{q}=0.08 \mathrm{~T}_{c}$ and $c_{g}=0.6$. (c) Lower panel: corresponding $g_{2}$ functions at different waiting times $t_{w}$.

have been put forward as possible explanations [56] underlying such unusual dynamics and subsequently extended to series of such intermittent events to account for both $q$ dependence of the relaxation rate and the KWW exponents $[57,58]$. The connection of these phenomenological models to the dynamics during an LLPS is not obvious, especially with regard to typical field theories such as the Cahn-Hilliard equation (CHE) used to model spinodal decomposition. A shared feature between colloidal gels and the LLPS, investigated here, is the presence of elastic deformations. These can arise in an LLPS from the viscoelastic properties, which are caused by a dynamical coupling of diffusion and stresses, and could explain the similarities between these observations [57]. In fact, the dynamical asymmetry of the two phases present in the system can lead to viscoelastic phase separation [59].

To obtain more insight into the underlying dynamical mechanisms, we compare the experimental data to numerical simulations of the temporal evolution of the $\mathrm{CHE}$ $[60,61]$ (here in 2D). The dynamics of the ordinary CHE shows faster dynamics for quenches to lower temperatures. As this is not what we observe in the experimental data, we introduce a dependence of the mobility $\mu$ on protein density $\rho(r, t)$, representing the slowdown of the dynamics when the density of the dense phase is increasing and its mobility freezes out [45] in the spirit of model C according to Ref. [2]. The parameter $c_{g}$ sets the concentration at which the mobility decreases (for details, see the SM). The simulations then yield a time dependent real space configuration $\rho(r, t)$ of the protein density [Fig. 4(c)], which is converted into an x-ray speckle pattern by means of its Fourier components $|\rho(q, t)|^{2}$ and analyzed with the same time correlation methods as applied to the experimental data $[45,46]$.

Figure 4(a) displays the temporal evolution of the spatially averaged mobility $\bar{\mu}\left(t_{w}\right)$ in the dense phase and
Fig. S10 the spatially averaged density $\bar{\rho}\left(t_{w}\right)$ of the dense and dilute phases for different values of $c_{g}$. A point in the 2D image was considered part of the dense phase if its concentration was higher than the initial concentration. The mobility of the dense phase drops quickly during the LLPS upon lowering the $c_{g}$ value. The densities in the dense and dilute phases do not reach their equilibrium values anymore, which is considered an arrest of the LLPS $[45,46]$.

Typical real space configurations are shown in Fig. 4(b) with the LLPS visible via the formation of domains of the diluted phase (blue) in a host matrix of the dense phase (red). The corresponding density profiles (see Fig. S9) show the typical hallmarks of the spinodal decomposition, with density fluctuations developing quickly and reaching rather smooth profiles at the end of the LLPS for a mobility that is independent of protein density and equal to 1 . In contrast, for $c_{g}=0.6$, we observe that the density fluctuations become immobile when reaching the threshold value, leading to smaller domains.

TTC and $g_{2}$ functions were computed following the procedure of experimental data. Figure 4(c) displays the TTC for the simulations and the corresponding $g_{2}$ functions. We identify a fast dynamic process during the early time of the LLPS, which represents the dynamics of spinodal interface formation between the dense and diluted phases. The dynamics quickly slows down with a sudden appearance of a second much slower relaxation process when the dense phase has formed. The relaxation times during the first stage are determined by the quench depth (smaller for deeper quenches) because the curvature of the free energy is here the main driving force for the velocity of interface formation. The corresponding $\mathrm{KWW}$ values $\gamma_{1}$ and $\gamma_{2}$ [Figs. S11(c) and S11(d)] are between 1.5 and 2.5, which is in good agreement with our experimental values.

Comparing the real space data and the parameters describing the dynamics, it is possible to see that the rise 
of the nonergodicity parameter starts during the onset of domain and coarsening dynamics. In this short transition time (see Fig. 3), domain coarsening is progressing in parallel with the final interface formation. After this transition, the domain coarsening is taking place. The dynamics seems to be composed of three processes: the coalescence of different domains, domain growth, and the spatial movement that is guided by the surface tension and precedes the coalescence of two domains. Its decorrelation time is larger for deeper quenches because of the lower transition concentration of the mobility [Fig. 4(c) and Fig. S11], representing a situation in which the temperature-dependent glass line of the dense host phase intersects the spinodal phase region. For the lower value of $c_{g}$, the diluted phase is essentially trapped inside the frozen host matrix [red in Fig. 4(b)].

Based on the simulation results, we can conclude that the kinetics of the systems is captured by model $\mathrm{C}$ as suggested before [2]. The dynamics, however, is only partially reproduced. While the slowdown of the late stage dynamics with increasing quench depth is correctly described, as well as the presence of two relaxation modes, model $\mathrm{C}$ does not reproduce the $\Gamma \propto q$ behavior observed in the experiment or the $q$ dependence of the KWW values of the slow dynamics (Fig. S11). We speculate that this is due to the neglect of the viscoelastic properties of the system in the CHE, which would lead to elastic deformation and motion inducing a linear dispersion relation.

In conclusion, we demonstrated the technique of low radiation dose XPCS and used it to study protein dynamics during an LLPS. The method delivers simultaneously information on the collective dynamics via XPCS and the structural evolution via the ensemble averaged scattering $I(q)$. The two-time correlation maps provide a high level of detail of the dynamics during a spinodal decomposition of Ig in solution with PEG. We identify distinctly different dynamical regimes of the LLPS with different temperature behaviors. The early stage dynamics reflects concentration fluctuation and interface formation and is faster for lower temperatures, reflecting stronger quench depths. In contrast, the later stage of coarsening is slower for lower temperatures, which is caused by the reduced mobility of the slowed down proteins comprising the host matrix. With simulations, we were able to identify a concentration and time dependence of the molecular-scale mobility that connects the dynamics of the condensate to molecular-scale quantities.

The authors acknowledge discussions with M. Oettel, financial support from the DFG and the BMBF (05K20VTA), and the allocation of beamtime by DESY. N. B. acknowledges the Alexander von Humboldt-Stiftung for a postdoctoral fellowship. A. R. acknowledges the Studienstiftung des Deutschen Volkes for a Ph.D. fellowship. C. G. acknowledges BMBF (Grants No. 05K19PS1 and No. 05K20PSA) for financial support.

*fajun.zhang@uni-tuebingen.de

†christian.gutt@uni-siegen.de

†rank.schreiber@uni-tuebingen.de; https://publons.com/ researcher/2502617/frank-schreiber/

[1] C. P. Brangwynne, C. R. Eckmann, D. S. Courson, A. Rybarska, C. Hoege, J. Gharakhani, F. Jülicher, and A. A. Hyman, Germline p granules are liquid droplets that localize by controlled dissolution/condensation, Science 324, 1729 (2009).

[2] J. Berry, C. P. Brangwynne, and M. Haataja, Physical principles of intracellular organization via active and passive phase transitions, Rep. Prog. Phys. 81, 046601 (2018).

[3] Y. Shin and C. P. Brangwynne, Liquid phase condensation in cell physiology and disease, Science 357, eaaf4382 (2017).

[4] A. A. Hyman and C. P. Brangwynne, Beyond stereospecificity: Liquids and mesoscale organization of cytoplasm, Dev. Cell 21, 14 (2011).

[5] L. Malinovska, S. Kroschwald, and S. Alberti, Protein disorder, prion propensities, and self-organizing macromolecular collectives, Biochim. Biophys. Acta Proteins Proteomics 1834, 918 (2013).

[6] S. C. Weber and C.P. Brangwynne, Getting RNA and protein in phase, Cell 149, 1188 (2012).

[7] E. Zaccarelli, Colloidal gels: Equilibrium and non-equilibrium routes, J. Phys. Condens. Matter 19, 323101 (2007).

[8] S. Manley, H. M. Wyss, K. Miyazaki, J. C. Conrad, V. Trappe, L. J. Kaufman, D. R. Reichman, and D. A. Weitz, Glasslike Arrest in Spinodal Decomposition as a Route to Colloidal Gelation, Phys. Rev. Lett. 95, 238302 (2005).

[9] J. C. Conrad, H. M. Wyss, V. Trappe, S. Manley, K. Miyazaki, L. J. Kaufman, A. B. Schofield, D. R. Reichman, and D. A. Weitz, Arrested fluid-fluid phase separation in depletion systems: Implications of the characteristic length on gel formation and rheology, J. Rheol. 54, 421 (2010).

[10] P. J. Lu, E. Zaccarelli, F. Ciulla, A. B. Schofield, F. Sciortino, and D. A. Weitz, Gelation of particles with short-range attraction, Nature (London) 453, 499 (2008).

[11] T. Gibaud and P. Schurtenberger, A closer look at arrested spinodal decomposition in protein solutions, J. Phys. Condens. Matter 21, 322201 (2009).

[12] T. Gibaud, F. Cardinaux, J. Bergenholtz, A. Stradner, and P. Schurtenberger, Phase separation and dynamical arrest for particles interacting with mixed potentials-The case of globular proteins revisited, Soft Matter 7, 857 (2011).

[13] F. Cardinaux, T. Gibaud, A. Stradner, and P. Schurtenberger, Interplay between Spinodal Decomposition and Glass Formation in Proteins Exhibiting Short-Range Attractions, Phys. Rev. Lett. 99, 118301 (2007).

[14] S. Da Vela, M. K. Braun, A. Dörr, A. Greco, J. Möller, Z. Fu, F. Zhang, and F. Schreiber, Kinetics of liquid-liquid phase separation in protein solutions exhibiting LCST phase behavior studied by time-resolved USAXS and VSANS, Soft Matter 12, 9334 (2016). 
[15] S. Da Vela, C. Exner, R. S. Schäufele, J. Möller, Z. Fu, F. Zhang, and F. Schreiber, Arrested and temporarily arrested states in a protein-polymer mixture studied by USAXS and VSANS, Soft Matter 13, 8756 (2017).

[16] B. Jiang, J. G. Tsavalas, and D. C. Sundberg, Water whitening of polymer films: Mechanistic studies and comparisons between water and solvent borne films, Progress in organic coatings 105, 56 (2017).

[17] S. Kim, J. Huang, Y. Lee, S. Dutta, H. Y. Yoo, Y. M. Jung, Y. M. Jho, H. Zeng, and D. S. Hwang, Complexation and coacervation of like-charged polyelectrolytes inspired by mussels, Proc. Natl. Acad. Sci. U.S.A. 113, E847 (2016).

[18] S. Wegmann, B. Eftekharzadeh, K. Tepper, K. M. Zoltowska, R. E. Bennett, S. Dujardin, P. R. Laskowski, D. MacKenzie, T. Kamath, and C. Commins, Tau protein liquidliquid phase separation can initiate tau aggregation, EMBO J. 37, e98049 (2018).

[19] H. Cai, B. Gabryelczyk, M. S. S. Manimekalai, G. Grber, S. Salentinig, and A. Miserez, Self-coacervation of modular squid beak proteins a comparative study, Soft Matter 13, 7740 (2017).

[20] A. S. Raut and D. S. Kalonia, Pharmaceutical perspective on opalescence and liquid-liquid phase separation in protein solutions, Mol. Pharmaceutics 13, 1431 (2016).

[21] A. Banc, J. Pincemaille, S. Costanzo, E. Chauveau, M.-S. Appavou, M.-H. Morel, P. Menut, and L. Ramos, Phase separation dynamics of gluten protein mixtures, Soft Matter 15, 6160 (2019).

[22] Y. Shin, J. Berry, N. Pannucci, M. P. Haataja, J. E. Toettcher, and C.P. Brangwynne, Spatiotemporal control of intracellular phase transitions using light-activated optodroplets, Cell 168, 159 (2017).

[23] A. R Strom, A. V Emelyanov, M. Mir, D. V. Fyodorov, X. Darzacq, and G. H. Karpen, Phase separation drives heterochromatin domain formation, Nature (London) 547, 241 (2017).

[24] S. Ambadipudi, J. Biernat, D. Riedel, E. Mandelkow, and M. Zweckstetter, Liquid-liquid phase separation of the microtubule-binding repeats of the Alzheimer-related protein tau, Nat. Commun. 8, 275 (2017).

[25] S. E. Reichheld, L. D. Muiznieks, F. W. Keeley, and S. Sharpe, Direct observation of structure and dynamics during phase separation of an elastomeric protein, Proc. Natl. Acad. Sci. U.S.A. 114, E4408 (2017).

[26] A. Boire, C. Sanchez, M.-H. Morel, M. P. Lettinga, and P. Menut, Dynamics of liquid-liquid phase separation of wheat gliadins, Sci. Rep. 8, 14441 (2018).

[27] Y. Gao, J. Kim, and M.E. Helgeson, Microdynamics and arrest of coarsening during spinodal decomposition in thermoreversible colloidal gels, Soft Matter 11, 6360 (2015).

[28] N. Begam, A. Ragulskaya, A. Girelli, H. Rahmann, S. F. Westermeier, M. Reiser, M. Sprung, F. Zhang, C. Gutt, and F. Schreiber, Kinetics of Network Formation and Heterogeneous Dynamics of an Egg White Gel Revealed by Coherent X-Ray Scattering, Phys. Rev. Lett. 126, 098001 (2021).

[29] A. Madsen, A. Fluerasu, and B. Ruta, Structural Dynamics of Materials Probed by X-Ray Photon Correlation Spectroscopy (Springer International Publishing, Cham, 2016), pp. 1617-1641.
[30] X. Lu, S. G. J. Mochrie, S. Narayanan, A. R. Sandy, and M. Sprung, How a Liquid Becomes a Glass Both on Cooling and On Heating, Phys. Rev. Lett. 100, 045701 (2008).

[31] Q. Zhang, D. Bahadur, E. M. Dufresne, P. Grybos, P. Kmon, R. L. Leheny, P. Maj, S. Narayanan, R. Szczygiel, S. Ramakrishnan, and A. Sandy, Dynamic Scaling of Colloidal Gel Formation at Intermediate Concentrations, Phys. Rev. Lett. 119, 178006 (2017).

[32] A. Madsen, L. L. Robert, G. Hongyu, S. Michael, and C. Orsolya, Beyond simple exponential correlation functions and equilibrium dynamics in $\mathrm{x}$-ray photon correlation spectroscopy, New J. Phys. 12, 055001 (2010).

[33] A. Malik, A. R. Sandy, L. B. Lurio, G. B. Stephenson, S. G. J. Mochrie, I. McNulty, and M. Sutton, Coherent X-Ray Study of Fluctuations During Domain Coarsening, Phys. Rev. Lett. 81, 5832 (1998).

[34] G. Brown, P. A. Rikvold, and M. Grant, Universality and scaling for the structure factor in dynamic order-disorder transitions, Phys. Rev. E 58, 5501 (1998).

[35] M. Grimaldo, F. Roosen-Runge, F. Zhang, F. Schreiber, and T. Seydel, Dynamics of proteins in solution, Q. Rev. Biophys. 52, 1 (2019).

[36] J. Moeller, M. Sprung, A. Madsen, and C. Gutt, X-ray photon correlation spectroscopy of protein dynamics at nearly diffraction-limited storage rings, IUCrJ 6, 794 (2019).

[37] J. Verwohlt, M. Reiser, L. Randolph, A. Matic, L. A. Medina, A. Madsen, M. Sprung, A. Zozulya, and C. Gutt, Low Dose X-Ray Speckle Visibility Spectroscopy Reveals Nanoscale Dynamics in Radiation Sensitive Ionic Liquids, Phys. Rev. Lett. 120, 168001 (2018).

[38] P. Vodnala, N. Karunaratne, L. Lurio, G. M. Thurston, M. Vega, E. Gaillard, S. Narayanan, A. Sandy, Q. Zhang, E. M. Dufresne, G. Foffi, P. Grybos, P. Kmon, P. Maj, and R. Szczygiel, Hard-sphere-like dynamics in highly concentrated alpha-crystallin suspensions, Phys. Rev. E 97, 020601 (R) (2018).

[39] C. M. Jeffries, M. A. Graewert, D. I. Svergun, and C. E. Blanchet, Limiting radiation damage for high-brilliance biological solution scattering: Practical experience at the embl p12 beamline PetraIII, J. Synchrotron Radiat. 22, 273 (2015).

[40] See Supplemental Material, which includes Refs. [41-44], at http://link.aps.org/supplemental/10.1103/PhysRevLett .126 .138004 for more detailed information on the simulation and the experiments as well as on fitting parameters.

[41] D. F. Griffiths and D. J. Higham, Numerical Methods for Ordinary Differential Equations: Initial Value Problems (Springer Science \& Business Media, New York, 2010).

[42] S. Puri and Y. Oono, Effect of noise on spinodal decomposition, J. Phys. A 21, L755 (1988).

[43] R. C Desai and R. Kapral, Dynamics of Self-Organized and Self-Assembled Structures (Cambridge University Press, Cambridge, England, 2009).

[44] K. Binder, Spinodal decomposition, in Systems Far from Equilibrium (Springer, New York, 1980), pp 76-90.

[45] D. Sappelt and J Jäckle, Spinodal decomposition with formation of a glassy phase, Europhys. Lett. 37, 13 (1997). 
[46] F. Sciortino, R. Bansil, H. E. Stanley, and P. Alstrm, Interference of phase separation and gelation: A zerothorder kinetic model, Phys. Rev. E 47, 4615 (1993).

[47] H. Furukawa, Dynamics-scaling theory for phase-separating unmixing mixtures: Growth rates of droplets and scaling properties of autocorrelation functions, Physica (Amsterdam) 123A, 497 (1984).

[48] O. Bikondoa, On the use of two-time correlation functions for x-ray photon correlation spectroscopy data analysis, J. Appl. Crystallogr. 50, 357 (2017).

[49] G. Williams and D. C. Watts, Non-symmetrical dielectric relaxation behaviour arising from a simple empirical decay function, Trans. Faraday Soc. 66, 80 (1970).

[50] W. van Megen, S. M. Underwood, and P. N. Pusey, Nonergodicity Parameters of Colloidal Glasses, Phys. Rev. Lett. 67, 1586 (1991).

[51] A. J. Wagner and J. M. Yeomans, Breakdown of Scale Invariance in the Coarsening of Phase-Separating Binary Fluids, Phys. Rev. Lett. 80, 1429 (1998).

[52] R. Bandyopadhyay, D. Liang, J. L. Harden, and R. L. Leheny, Slow dynamics, aging, and glassy rheology in soft and living matter, Solid State Commun. 139, 589 (2006).

[53] A. Jain, F. Schulz, I. Lokteva, L. Frenzel, G. Grbel, and F. Lehmkhler, Anisotropic and heterogeneous dynamics in an aging colloidal gel, Soft Matter 16, 2864 (2020).

[54] P. Ballesta, A. Duri, and L. Cipelletti, Unexpected drop of dynamical heterogeneities in colloidal suspensions approaching the jamming transition, Nat. Phys. 4, 550 (2008).
[55] Z. W. Wu, W. Kob, W.-H. Wang, and L. Xu, Stretched and compressed exponentials in the relaxation dynamics of a metallic glass-forming melt, Nat. Commun. 9, 5334 (2018).

[56] L. Cipelletti, S. Manley, R. C. Ball, and D. A. Weitz, Universal Aging Features in the Restructuring of Fractal Colloidal Gels, Phys. Rev. Lett. 84, 2275 (2000).

[57] A. Duri and L. Cipelletti, Length scale dependence of dynamical heterogeneity in a colloidal fractal gel, Europhys. Lett. 76, 972 (2006).

[58] C. Caronna, Y. Chushkin, A. Madsen, and A. Cupane, Dynamics of Nanoparticles in a Supercooled Liquid, Phys. Rev. Lett. 100, 055702 (2008).

[59] H. Tanaka and Y. Nishikawa, Viscoelastic Phase Separation of Protein Solutions, Phys. Rev. Lett. 95, 078103 (2005).

[60] J. W. Cahn, Phase separation by spinodal decomposition in isotropic systems, J. Chem. Phys. 42, 93 (1965).

[61] J. W. Cahn and J. E. Hilliard, Free energy of a nonuniform system. i. interfacial free energy, J. Chem. Phys. 28, 258 (1958).

[62] D. M. Mitrea and R. W. Kriwacki, Phase separation in biology; functional organization of a higher order, Cell Commun. Signal 14, 1 (2016).

[63] J. A. Thomson, P. Schurtenberger, G. M. Thurston, and G. B. Benedek, Binary liquid phase separation and critical phenomena in a protein/water solution, Proc. Natl. Acad. Sci. U.S.A. 84, 7079 (1987). 